\section{Utilização de eritropoetina por pacientes incidentes em hemodiálise no Sistema Único de Saúde, Brasil, 2002-2003}

\author{
Erythropoietin use by incident hemodialysis \\ patients in the Brazilian Unified National \\ Health System, 2002-2003
}

Thaís Caroline Gurgel 1 Mariângela Leal Cherchiglia 1 Francisco de Assis Acurcio 1 Daniele Araújo Campos Szuster 1 Isabel Cristina Gomes 2 Eli Iola Gurgel Andrade 1

\section{Introdução}

\footnotetext{
1 Faculdade de Medicina, Universidade Federal de Minas Gerais, Belo Horizonte, Brasil.

2 Instituto de Ciências Exatas, Universidade Federal de Minas Gerais, Belo Horizonte, Brasil.

Correspondência T. C. Gurgel Faculdade de Medicina, Universidade Federal de Minas Gerais.

Rua Walter Pedro Lloyd 2 , Nova Lima, MG 34000-000, Brasil.

thaiscarolgurgel@bol.com.br
}

\section{Abstract}

This study aimed to describe the demographic and epidemiological profile of Brazilian patients entering hemodialysis from 2002 to 2003 and identify predictors of erythropoietin use. The study analyzed demographic and clinical characteristics and dialysis facility-related variables from 32,136 patients identified by deterministic-probabilistic matching in the database of authorizations for high-cost procedures and the Mortality Information System. Poisson regression was used to identify predictors of erythropoietin use. Male gender, age < 65 years, diabetic renal failure, arteriovenous fistula at the beginning of hemodialysis, and living in States of Brazil other than Mato Grosso were predictors of erythropoietin use. The policy of care for chronic kidney disease, resource allocation for States and municipalities, and anemia management according to the patient's drug therapy profile need to be revised in order to reduce observed inequities in erythropoietin use.

Erythropoietin; Renal Dialysis; Anemia; Equity in Access
A doença renal crônica é uma síndrome metabólica decorrente de uma injúria renal inicial, seguida de perda lenta, progressiva e irreversível das funções glomerular, tubular e endócrina dos rins 1,2. Entre as causas mais comuns, citam-se hipertensão arterial, diabetes mellitus, glomerulonefrites e doenças autoimunes. Os fatores de risco que podem acelerar o progresso da doença são tabagismo, obesidade e dislipidemias 2,3 . No estágio mais avançado, comumente chamado doença renal em estágio final, os rins não conseguem mais manter o equilíbrio hidroeletrolítico do organismo, sendo necessária uma das modalidades das terapias renais substitutivas - hemodiálise, diálise peritoneal ou transplante renal para garantir a sobrevivência do paciente 1,2 .

Uma importante complicação da doença renal crônica é a anemia. Decorre da diminuição da sobrevida das hemácias nos pacientes e, também, da perda progressiva da capacidade de síntese renal do hormônio eritropoetina, que estimula a produção dos eritrócitos pela medula óssea 4,5 .

A anemia pode ocorrer em qualquer estágio da doença renal crônica, tornando-se mais prevalente e severa à medida que a doença progride. Infelizmente, no Brasil, há poucos dados sobre a prevalência desse tipo de anemia. A maioria dos estudos fornece estimativas indi- 
retas do panorama da anemia em nosso país, relacionando-se, por exemplo, ao percentual de utilização de medicamentos para tratar anemia em pacientes em tratamento dialítico 3,6 , ou à investigação de variações nos níveis de hemoglobina em pacientes tratados 7. Dados do Censo de Diálise de 20083 apontaram um percentual de $83 \%$ de pacientes em uso de medicamento para tratar anemia e um percentual de $41,7 \%$ dos pacientes tratados com exames de hemoglobina abaixo do alvo terapêutico. Por sua vez, o Ministério da Saúde 6 estima, com base em informações do Sistema de Informações Ambulatoriais (SIA) referentes a 2008 e 2009, que cerca de $80 \%$ dos pacientes em hemodiálise no período utilizaram medicamento para tratar anemia.

Por outro lado, um estudo em pacientes adultos nos Estados Unidos encontrou prevalência global da anemia na doença renal crônica de aproximadamente $47 \%$, variando de $26,7 \%$, em pacientes com doença renal crônica em estágio inicial, a 75,5\%, naqueles com doença renal crônica em estágio final, em tratamento pré-dialítico ${ }^{8}$. Segundo outro estudo norte-americano, cerca de $40 \%$ dos pacientes, antes mesmo da progressão para doença renal em estágio final, possuem hemoglobina menor que $11 \mathrm{~g} / \mathrm{dL}$ e, nos pacientes com doença renal em estágio final, a anemia é um achado praticamente universal 9. Além disso, nos pacientes com doença renal em estágio final submetidos à hemodiálise, ocorre perda sanguínea adicional, decorrente do processamento do sangue pela máquina de hemodiálise, tornando a anemia mais severa nesses casos 10,11.

Sem tratamento, a anemia pode causar fadiga, perda da capacidade cognitiva e física, além de predispor a quedas e a fraturas 7 . Também pode ocasionar aumento da sobrecarga cardíaca, levando à hipertrofia ventricular esquerda e à miocardiopatia 6,8. Assim, a anemia em pacientes com doença renal crônica está associada ao aumento da morbidade e da mortalidade, à progressão mais rápida para doença renal em estágio final e à piora de sua qualidade de vida 6,12,13 .

O tratamento padrão para a anemia na doença renal crônica, atualmente, consiste na administração de agentes estimulantes da eritropoiese 4,7,10. Dentre estes, por seu custo mais baixo, a eritropoetina humana recombinante, ou alfaepoetina, é o fármaco mais utilizado na terapêutica, inclusive no Brasil 4,7,10,14.

O Sistema Único de Saúde (SUS) disponibiliza a eritropoetina gratuitamente. Sendo um medicamento do Componente Especializado de Assistência Farmacêutica, nos termos da Portaria GM/MS $n^{\circ}$. 2.981/2009 6,14, é financiada pelo Ministério da Saúde, com cofinanciamento pe- las Secretarias Estaduais de Saúde. Estas também são responsáveis pela aquisição, distribuição e dispensação do medicamento ao usuário final 14 .

O fornecimento aos pacientes baseia-se no Protocolo Clínico e nas Diretrizes Terapêuticas para o Tratamento da Anemia na Insuficiência Renal Crônica, estabelecidos por meio da Portaria SAS/MS no. 226/2010 6. O objetivo do tratamento, de acordo com o protocolo, é manter a hemoglobina do paciente entre $11 \mathrm{~g} / \mathrm{dL}$ e $12 \mathrm{~g} /$ $\mathrm{dL}$, e o hematócrito entre $33 \%$ e $36 \%$. Esses alvos terapêuticos também são preconizados pelo European Renal Association's Best Practice Guidelines (EBPG) e pelo Dialysis Outcomes Quality Initiative of the US National Kidney Foundation (NKF-DOQI) 15,16. Níveis de hemoglobina acima de $12 \mathrm{~g} / \mathrm{dL}$, de um modo geral, não são recomendados, pois há risco de propiciar a ocorrência de eventos cardiovasculares e de aumentar o risco de morte nos pacientes em tratamento dialítico 6,15,16.

Complementarmente, deve haver o monitoramento dos estoques de ferro no organismo, para verificar a necessidade de suplementação do mineral. Esta é feita com o objetivo de manter a ferritina sérica e a transferrina em níveis adequados para que a eritropoiese, estimulada pela eritropoetina, ocorra de maneira efetiva. Espera-se que o tratamento da anemia melhore a qualidade de vida dos pacientes, diminua o número de hospitalizações, reduza a necessidade de transfusões sanguíneas, previna ou melhore a hipertrofia ventricular esquerda e diminua a morbimortalidade 6,10,15.

Mediante um estudo sobre utilização de medicamentos de alto custo no Brasil, disponibilizados majoritariamente pelo SUS, no período entre 2000 e 2004, verificou-se que a eritropoetina foi o mais utilizado 17 . A hemodiálise é a modalidade predominante na terapia renal substitutiva no Brasil 3,6,18 e, também, a que apresenta percentual mais elevado de pacientes em uso de eritropoetina, em virtude da maior severidade da anemia nestes pacientes 11,19 .

Considerando os princípios doutrinários do SUS, no que diz respeito à equidade no acesso aos cuidados de saúde e às diretrizes estabelecidas pela Política Nacional de Medicamentos, este estudo buscou descrever o perfil demográfico e epidemiológico dos pacientes incidentes em hemodiálise que utilizaram eritropoetina nos anos de 2002 e 2003, no Brasil. Objetivou, ainda, compará-los, no mesmo período, com aqueles incidentes em hemodiálise que não utilizaram o medicamento e, finalmente, identificar fatores associados ao uso de eritropoetina, conforme as variáveis que caracterizam os pacientes. 


\section{Métodos}

Este estudo é parte do Projeto TRS - Avaliação Econômico-Epidemiológica das Terapias Renais Substitutivas no Brasil, apoiado pelo Ministério da Saúde. Trata-se de um estudo transversal prospectivo não concorrente, que utilizou dados da Base Nacional em Terapias Renais Substitutivas 2000-2004, desenvolvida pelo Grupo de Pesquisa em Economia da Saúde da Universidade Federal de Minas Gerais (UFMG). Essa base foi construída utilizando-se dados do subsistema de Autorização de Procedimentos de Alto Custo (APAC) do SAI, do Sistema de Informações sobre Mortalidade (SIM) e do Sistema de Informações Hospitalares (SIH), referentes ao período de 2000 a 2004, por meio do método de linkage probabilístico-determinístico 20.

A coorte incidente da Base Nacional em Terapia Renal Substitutiva no período de 2000 a 2004 era composta por 90.356 pacientes. Foram considerados elegíveis para o estudo os pacientes incidentes na modalidade hemodiálise nos anos 2002 e 2003, com idade igual ou superior a 18 anos (32.459). Foram excluídos 323 pacientes que tinham registro de procedimento específico para tratamento de HIV. A coorte final foi composta de 32.136 pacientes.

As seguintes variáveis foram selecionadas para investigação:

Variáveis demográficas: sexo; idade; região de residência no primeiro registro observado; unidade federativa de residência no primeiro registro observado; Índice de Desenvolvimento Humano (IDH) do município de residência do paciente no primeiro registro observado. $\mathrm{O}$ IDH é um indicador composto que engloba três dimensões: renda, educação e esperança média de vida 21 ; tendo sido categorizado como baixo (IDH entre 0 a 0,499), médio (IDH entre 0,500 e 0,799) e alto (IDH entre 0,800 e 1,0).

Variáveis clínicas: causa primária de doença renal crônica; uso de eritropoetina; suplementação de ferro III durante o período de observação; realização de transplante renal durante o período de observação; tempo de acompanhamento; situação ao fim do estudo - censura ou óbito; tempo de acompanhamento até o óbito.

Variáveis relacionadas à unidade de diálise: ano de entrada em hemodiálise; presença de fístula arteriovenosa ao iniciar hemodiálise; natureza jurídica da unidade prestadora - pública ou privada.

Fez-se análise estatística descritiva, valendose de proporções, para variáveis categóricas, e de medidas de tendência central e de dispersão, para variáveis contínuas. Os estados do Acre, Amapá e Roraima, que apresentaram o menor número de pacientes, cada um deles com menos de 100, foram agrupados como subgrupo único, denominado "outros estados" (Tabela 1). Com objetivo de identificar possíveis diferenças significantes entre os grupos de pacientes, as análises comparativas foram feitas segundo o uso de eritropoetina. Para a comparação de médias entre os dois grupos, em relação às variáveis contínuas, foi utilizado o teste não paramétrico de MannWhitney, assumindo-se variâncias desiguais. O teste qui-quadrado de Pearson foi utilizado para comparar proporções entre os grupos, em relação às variáveis categóricas. A decisão estatística foi tomada considerando-se o nível de significância de 5\%. O programa estatístico utilizado na análise foi o SPSS 16.0 (SPSS Inc., Chicago, Estados Unidos).

Para identificação dos fatores associados ao uso de eritropoetina, foi proposto um modelo múltiplo de regressão de Poisson com variância robusta. A variável dependente foi o uso de eritropoetina. As covariáveis propostas para compor o modelo múltiplo de regressão de Poisson foram as mesmas usadas para caracterizar os pacientes. Procedeu-se à análise univariada adotando-se o nível de significância de $20 \%$. Para inclusão das variáveis no modelo múltiplo, foi utilizado o método forward. O modelo final foi composto das seguintes variáveis: sexo; grupos etários; unidade federativa de residência no primeiro registro observado; presença de diabetes como causa de doença renal crônica; presença de fístula ao iniciar hemodiálise. Nesta etapa da análise, a decisão estatística foi tomada adotando-se o nível de significância de 5\%. O software estatístico utilizado na construção do modelo múltiplo foi o R 2.7.2 (The R Foundation for Statistical Computing, Viena, Áustria; http://www.r-project.org).

O Projeto TRS - Avaliação Econômico-Epidemiológica das Terapias Renais Substitutivas no Brasil foi aprovado pelo Comitê de Ética e Pesquisa da UFMG através do parecer ETIC 397-04.

\section{Resultados}

As características da população estudada estão descritas na Tabela 1. Com relação à coorte geral, a maioria dos pacientes era do sexo masculino, concentrava-se no grupo etário dos 45 a 64 anos e residia na Região Sudeste no início do tratamento. As regiões Norte e Centro-oeste tiveram as menores proporções de pacientes. O Estado de São Paulo concentrou o maior número de pacientes, a maioria dos quais residia em municípios com IDH médio ou alto.

Uma pequena proporção de pacientes (11\%) iniciou hemodiálise com fístula arteriovenosa. 


\section{Tabela 1}

Características demográficas, clínicas e relacionadas à unidade de diálise dos pacientes do Sistema Único de Saúde (SUS) incidentes em hemodiálise, segundo o uso de eritropoetina, Brasil, 2002 e 2003.

\begin{tabular}{|c|c|c|c|c|c|c|}
\hline & \multicolumn{4}{|c|}{ Uso de eritropoetina } & \multicolumn{2}{|c|}{ Total } \\
\hline & \multicolumn{2}{|c|}{ Sim } & \multicolumn{2}{|c|}{ Não } & \multirow[b]{2}{*}{$\mathbf{n}$} & \multirow[b]{2}{*}{$\%$} \\
\hline & $\mathrm{n}$ & $\%$ & $\mathrm{n}$ & $\%$ & & \\
\hline Total & 19.298 & 60 & 12.838 & 40 & 32.136 & 100 \\
\hline \multicolumn{7}{|l|}{ Variável } \\
\hline \multicolumn{7}{|l|}{ Sexo } \\
\hline Masculino & 7.318 & 57 & 11.168 & 58 & 18.486 & 57 \\
\hline Feminino & 5.520 & 43 & 8.130 & 42 & 13.650 & 43 \\
\hline \multicolumn{7}{|l|}{ Idade à entrada em hemodiálise (anos) * } \\
\hline Média (desvio padrão) & $52(16)$ & & $58(16)$ & & $55(16)$ & \\
\hline Mediana & 53 & & 59 & & 56 & \\
\hline \multicolumn{7}{|c|}{ Grupo etário à entrada em hemodiálise (anos) * } \\
\hline $18-44$ & 6.156 & 32 & 2.738 & 21 & 8.894 & 28 \\
\hline $45-64$ & 8.702 & 45 & 5.360 & 42 & 14.062 & 43 \\
\hline$\geq 65$ & 4.440 & 23 & 4.740 & 37 & 9.180 & 29 \\
\hline \multicolumn{7}{|l|}{ Região de residência no 1 o registro * } \\
\hline Sudeste & 10.175 & 53 & 5.317 & 42 & 15.477 & 49 \\
\hline Nordeste & 4.889 & 25 & 3.276 & 25 & 8.153 & 25 \\
\hline Centro-oeste & 1.007 & 5 & 658 & 5 & 1.665 & 5 \\
\hline Sul & 2.169 & 11 & 3.110 & 24 & 5.268 & 16 \\
\hline Norte & 1.068 & 6 & 528 & 4 & 1.573 & 5 \\
\hline \multicolumn{7}{|c|}{ Unidade Federativa de residência no 1ㅇ registro * } \\
\hline Alagoas & 495 & 3 & 404 & 3 & 899 & 3 \\
\hline Amazonas & 290 & 2 & 50 & 0 & 340 & 1 \\
\hline Bahia & 1.090 & 6 & 622 & 5 & 1.712 & 5 \\
\hline Ceará & 628 & 3 & 468 & 4 & 1.096 & 3 \\
\hline Distrito Federal & 304 & 2 & 73 & 0 & 377 & 1 \\
\hline Espírito Santo & 438 & 2 & 176 & 1 & 614 & 2 \\
\hline Goiás & 548 & 3 & 156 & 1 & 704 & 2 \\
\hline Maranhão & 383 & 2 & 226 & 2 & 609 & 2 \\
\hline Minas Gerais & 2.092 & 11 & 1.623 & 13 & 3.715 & 12 \\
\hline Mato Grosso & 49 & 0 & 319 & 3 & 368 & 1 \\
\hline Mato Grosso do Sul & 106 & 1 & 110 & 1 & 216 & 0 \\
\hline Pará & 415 & 2 & 152 & 1 & 567 & 2 \\
\hline Paraíba & 538 & 3 & 382 & 3 & 920 & 3 \\
\hline Paraná & 1.180 & 6 & 599 & 5 & 1.779 & 6 \\
\hline Pernambuco & 955 & 5 & 608 & 5 & 1.563 & 5 \\
\hline Piauí & 223 & 1 & 231 & 2 & 454 & 1 \\
\hline Rio de Janeiro & 1.792 & 9 & 1.621 & 13 & 3.413 & 11 \\
\hline Rio Grande do Norte & 384 & 2 & 267 & 2 & 651 & 2 \\
\hline Rio Grande do Sul & 406 & 2 & 2.060 & 16 & 2.466 & 8 \\
\hline Rondônia & 143 & 1 & 125 & 1 & 268 & 1 \\
\hline Santa Catarina & 583 & 3 & 440 & 3 & 1.023 & 3 \\
\hline São Paulo & 5.853 & 30 & 1.882 & 15 & 7.735 & 24 \\
\hline Sergipe & 191 & 1 & 58 & 0 & 249 & 1 \\
\hline Tocantins & 102 & 0 & 142 & 1 & 244 & 1 \\
\hline Outros (Acre + Amapá + Roraima) & 110 & 0 & 44 & 0 & 154 & 0 \\
\hline
\end{tabular}

(continua) 


\begin{tabular}{|c|c|c|c|c|c|c|}
\hline & \multicolumn{4}{|c|}{ Uso de eritropoetina } & \multicolumn{2}{|c|}{ Total } \\
\hline & \multicolumn{2}{|c|}{ Sim } & \multicolumn{2}{|c|}{ Não } & \multirow[b]{2}{*}{$\mathrm{n}$} & \multirow[b]{2}{*}{$\%$} \\
\hline & $\mathrm{n}$ & $\%$ & $\mathrm{n}$ & $\%$ & & \\
\hline \multicolumn{7}{|l|}{ IDH do município de residência no 1ㅇ registro * } \\
\hline Baixo & 6 & 0 & 2 & 0 & 8 & 0 \\
\hline Médio & 10.170 & 53 & 7.280 & 57 & 17.450 & 54 \\
\hline Alto & 9.122 & 47 & 5.551 & 43 & 14.673 & 46 \\
\hline \multicolumn{7}{|c|}{ Presença de fístula arteriovenosa à entrada em hemodiálise * } \\
\hline Sim & 2.406 & 13 & 1.123 & 9 & 3.529 & 11 \\
\hline Não & 16.892 & 87 & 11.715 & 91 & 28.607 & 89 \\
\hline \multicolumn{7}{|l|}{ Causa da doença renal crônica no 1 o registro * } \\
\hline Diabetes mellitus/Complicações renais & 2.976 & 16 & 1.854 & 15 & 4.830 & 15 \\
\hline Glomerulonefrites & 1.901 & 10 & 953 & 7 & 2.854 & 9 \\
\hline Hipertensão e outras doenças cardiovasculares & 4.513 & 23 & 3.001 & 23 & 7.514 & 23 \\
\hline Indeterminada & 8.748 & 45 & 6.061 & 47 & 14.809 & 46 \\
\hline Outras doenças & 1.160 & 6 & 969 & 8 & 2.129 & 7 \\
\hline \multicolumn{7}{|l|}{ Suplementação de ferro III no período * } \\
\hline Sim & 4.876 & 25 & 741 & 6 & 5.617 & 18 \\
\hline Não & 14.422 & 75 & 12.097 & 94 & 26.519 & 82 \\
\hline \multicolumn{7}{|l|}{ Realização de transplante renal no período * } \\
\hline Sim & 1.263 & 6 & 343 & 3 & 1.606 & 5 \\
\hline Não & 18.035 & 94 & 12.495 & 97 & 30.530 & 95 \\
\hline \multicolumn{7}{|l|}{ Óbito durante o período de observação * } \\
\hline $\operatorname{Sim}$ & 5.088 & 26 & 8.514 & 66 & 13.602 & 42 \\
\hline Não & 14.210 & 74 & 4.324 & 34 & 18.534 & 58 \\
\hline \multicolumn{7}{|l|}{ Tempo de acompanhamento do estudo (meses) * } \\
\hline Média (desvio padrão) & $20(9)$ & & $10(10)$ & & $16(11)$ & \\
\hline Mediana & 20 & & 5 & & 16 & \\
\hline \multicolumn{7}{|l|}{ Tempo de acompanhamento até óbito (meses) * } \\
\hline Média (desvio padrão) & $13(8)$ & & $5(5)$ & & $8(8)$ & \\
\hline Mediana & 11 & & 3 & & 5 & \\
\hline \multicolumn{7}{|l|}{ Ano de entrada em hemodiálise * } \\
\hline 2002 & 9.711 & 50 & 5.966 & 47 & 15.677 & 49 \\
\hline 2003 & 9.587 & 50 & 6.842 & 53 & 16.429 & 51 \\
\hline \multicolumn{7}{|l|}{ Natureza jurídica da unidade de diálise } \\
\hline Privada & 12.999 & 68 & 7.746 & 61 & 20.745 & 65 \\
\hline Pública & 4.519 & 23 & 2.757 & 21 & 7.276 & 23 \\
\hline Ignorada & 1.780 & 9 & 2.335 & 18 & 4.115 & 12 \\
\hline
\end{tabular}

* Valor de $p<0,001$.

Houve elevado percentual de pacientes com causa primária de doença renal crônica indeterminada (46\%). Dentre as causas determinadas, hipertensão e outras doenças cardiovasculares (23\%) tiveram a maior proporção, sendo seguidas pelo diabetes mellitus (15\%).

Dos pacientes analisados, $60 \%$ fizeram uso de eritropoetina durante o tempo do estudo e $18 \%$ fizeram suplementação de ferro III no período. Apenas 5\% realizaram transplante renal durante o tempo de acompanhamento e $42 \%$ haviam evoluído para o óbito ao fim do estudo. Tanto o tempo médio quanto o tempo mediano de acompanhamento foram de 16 meses. Com relação ao ano de entrada para a coorte global houve proporções semelhantes de pacientes ingressando em hemodiálise em 2002 e 2003.

Comparando-se os pacientes que utilizaram eritropoetina com os que não utilizaram tal medicamento, percebeu-se que as proporções entre os sexos foram semelhantes nos dois grupos. Os pacientes que utilizaram eritropoetina, porém, 
tiveram menor média e mediana de idade e se concentraram nos grupos etários inferiores a 65 anos. Para ambos os grupos, a maior concentração de pacientes esteve no Sudeste, entretanto a distribuição destes entre as regiões foi bastante distinta para os dois grupos. A Região Sul mostrou um percentual importante de pacientes no grupo que não utilizou eritropoetina. Em termos absolutos, São Paulo foi o estado onde residia a maior parte dos pacientes que utilizaram eritropoetina, seguido de Minas Gerais, Rio de Janeiro e Bahia (Tabela 1). Contudo, em termos relativos, o Estado do Amazonas deteve a maior proporção de pacientes utilizando eritropoetina, seguido do Distrito Federal, Goiás, Sergipe e São Paulo (Figura 1). O Estado do Rio Grande do Sul apresentou o maior número absoluto de pacientes que não utilizaram o medicamento, seguido de São Paulo, Minas Gerais e Rio de Janeiro (Tabela 1). Em termos relativos, o Estado do Mato Grosso apresentou o menor percentual de pacientes utilizando eritropoetina, seguido do Rio Grande do Sul, Tocantins, Piauí e Mato Grosso do Sul (Figura 2). Para ambos os grupos, a maioria dos pacientes residia em municípios com IDH médio ou alto, a exemplo do observado para a coorte geral (Tabela 1).

Em relação ao ano de entrada em hemodiálise, o grupo de pacientes que utilizou eritropoetina apresentou distribuição mais homogênea de pacientes, em relação ao grupo que não utilizou o medicamento, entre os dois anos de entrada pesquisados. O primeiro grupo também teve maior percentual de pacientes que iniciaram hemodiálise com fístula. Com relação à causa primária de doença renal crônica, apresentou maiores percentuais de diabetes e de glomerulonefrites, mantendo, para as outras causas, proporções semelhantes às encontradas no grupo que não utilizou o medicamento. Aquele também teve maior percentual de pacientes que fizeram suplementação de ferro III e que realizaram transplante, durante o período de acompanhamento. A proporção de óbitos no grupo que utilizou eritropoetina foi quase um terço daquela observada no grupo que não utilizou o medicamento. O tempo de acompanhamento até o óbito foi maior para o grupo que utilizou eritropoetina. Além disso, o tempo de acompanhamento durante o estudo no grupo de pacientes utilizando eritropoetina foi o dobro daquele observado no grupo de pacientes que não utilizaram o fármaco.

Em relação à natureza jurídica da unidade de diálise, as distribuições dos pacientes entre as categorias foram semelhantes, com ambos os grupos apresentando maior concentração em unidades de diálise privadas.
Por meio da comparação entre os grupos (Tabela 1), foram encontradas diferenças estatisticamente significativas em relação a todas as variáveis, com exceção de sexo e natureza jurídica da unidade de diálise.

Os fatores associados a uma maior probabilidade de uso de eritropoetina foram: sexo masculino; idade inferior a 65 anos; presença de diabetes como causa primária de doença renal crônica; presença de fístula arteriovenosa ao iniciar hemodiálise; residir em outras Unidades Federativas em relação ao Mato Grosso. Dentre todas as unidades federativas, apenas para o Rio Grande do Sul, a razão de prevalência de uso de eritropoetina maior em relação ao Mato Grosso não assumiu significância estatística (Tabela 2).

\section{Discussão}

Na coorte estudada, o percentual encontrado de $60 \%$ de pacientes utilizando eritropoetina está aquém do esperado, considerando que a anemia é uma complicação quase universal em pacientes com doença renal em estágio final 9. Dados do Censo de Diálise de 20083 mostraram que aproximadamente $83 \%$ dos pacientes em tratamento dialítico, nas unidades que responderam ao censo, utilizaram eritropoetina. Estudando pacientes no Reino Unido, Richardson et al. 11 relataram utilização de agentes estimulantes da eritropoiese por cerca de $88 \%$ dos pacientes em hemodiálise. Pérez-García et al. ${ }^{22}$, em um estudo com pacientes incidentes em hemodiálise na Espanha, relataram utilização de eritropoetina por $80 \%$ da população do estudo. O resultado encontrado neste estudo pode significar subtratamento dos pacientes com doença renal em estágio final, incidentes em hemodiálise.

No entanto, vale ressaltar que os resultados deste estudo se referem ao período de 2002 a 2003, momento em que o protocolo de tratamento da anemia na doença renal crônica estava recém-implantado no Brasil. A implantação dessa norma e a publicação da Portaria GM/MS $n^{o}$. $1.318 / 200223$, regulamentando o fornecimento da eritropoetina exclusivamente mediante critérios definidos no protocolo, podem ter modificado os padrões de prescrição e de utilização da eritropoetina, embora o presente estudo não seja capaz de medir o impacto da publicação desses marcos regulatórios.

Por terem sido analisados dados provenientes de bases administrativas, utilizadas com finalidade de cobrança e pagamento de procedimentos, devem-se considerar aspectos da execução e do gerenciamento dos "medicamentos de alto 
Figura 1

Distribuição dos pacientes, segundo o uso de eritropoetina, nos estados com maior percentual de utilização do medicamento.

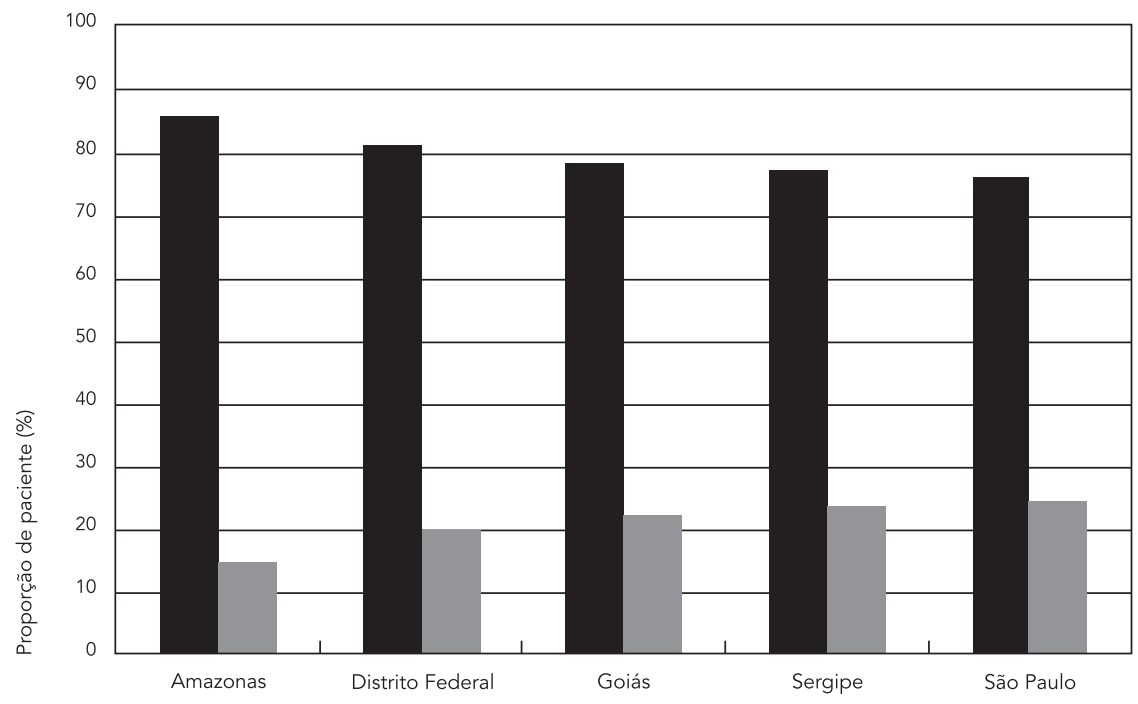

Fonte: Base Nacional em Terapias Renais Substitutivas.

Figura 2

Distribuição dos pacientes, segundo o uso de eritropoetina, nos estados com menor percentual de utilização do medicamento.

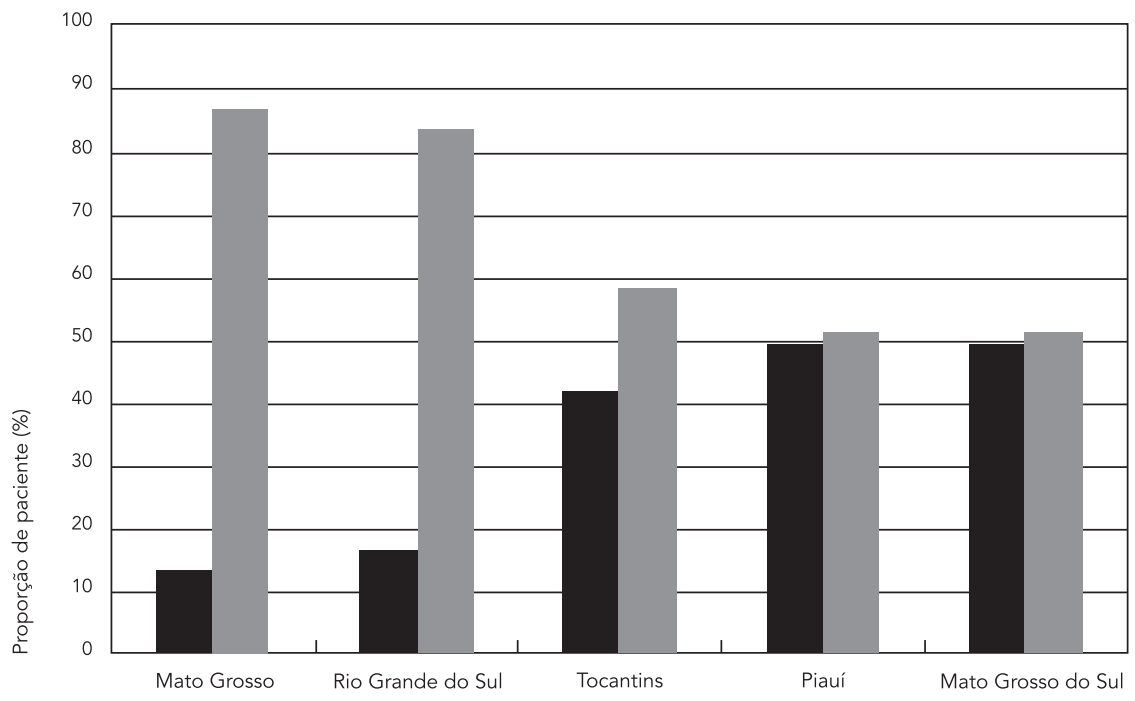

Fonte: Base Nacional em Terapias Renais Substitutivas. 
Modelo múltiplo de regressão de Poisson para fatores associados ao uso de eritropoetina, em pacientes incidentes em hemodiálise no Brasil, 2002 e 2003.

\begin{tabular}{|c|c|c|c|}
\hline Variável & RP & IC95\% & Valor de $\mathrm{p}$ \\
\hline \multicolumn{4}{|l|}{ Sexo } \\
\hline Feminino & 1,000 & & \\
\hline Masculino & 1,022 & 1,$005 ; 1,039$ & 0,013 \\
\hline \multicolumn{4}{|l|}{ Idade (anos) } \\
\hline$\geq 65$ & 1,000 & & \\
\hline $18-44$ & 1,385 & 1,$352 ; 1,419$ & $<0,0001$ \\
\hline $45-64$ & 1,238 & 1,$209 ; 1,267$ & $<0,0001$ \\
\hline \multicolumn{4}{|l|}{ Diabetes } \\
\hline Não & 1,000 & & \\
\hline Sim & 1,063 & 1,$039 ; 1,088$ & $<0,0001$ \\
\hline \multicolumn{4}{|l|}{ Fístula ao iniciar hemodiálise } \\
\hline Não & 1,000 & & \\
\hline $\operatorname{Sim}$ & 1,140 & 1,$114 ; 1,166$ & $<0,0001$ \\
\hline \multicolumn{4}{|c|}{ Unidade Federativa de residência no 1ㅇ registro } \\
\hline Mato Grosso & 1,000 & & \\
\hline Alagoas & 4,097 & 3,$135 ; 5,353$ & $<0,0001$ \\
\hline Amazonas & 6,335 & 4,$861 ; 8,255$ & $<0,0001$ \\
\hline Bahia & 4,717 & 4,$624 ; 6,138$ & $<0,0001$ \\
\hline Ceará & 4,277 & 3,$279 ; 5,580$ & $<0,0001$ \\
\hline Distrito Federal & 5,990 & 4,$592 ; 7,813$ & $<0,0001$ \\
\hline Espírito Santo & 5,405 & 4,$143 ; 7,050$ & $<0,0001$ \\
\hline Goiás & 5,728 & 4,$399 ; 7,458$ & $<0,0001$ \\
\hline Maranhão & 4,700 & 3,$596 ; 6,143$ & $<0,0001$ \\
\hline Minas Gerais & 4,209 & 3,$237 ; 5,473$ & $<0,0001$ \\
\hline Mato Grosso do Sul & 3,741 & 2,$794 ; 5,009$ & $<0,0001$ \\
\hline Pará & 5,526 & 4,$237 ; 7,207$ & $<0,0001$ \\
\hline Paraíba & 4,495 & 3,$443 ; 5,867$ & $<0,0001$ \\
\hline Pernambuco & 4,638 & 3,$562 ; 6,038$ & $<0,0001$ \\
\hline Piauí & 3,625 & 2,$747 ; 4,786$ & $<0,0001$ \\
\hline Paraná & 4,952 & 3,$806 ; 6,443$ & $<0,0001$ \\
\hline Rio de Janeiro & 3,968 & 3,$051 ; 5,162$ & $<0,0001$ \\
\hline Rio Grande do Norte & 4,422 & 3,$380 ; 5,784$ & $<0,0001$ \\
\hline Rio Grande do Sul & 1,277 & 0,$969 ; 1,682$ & 0,085 \\
\hline Rondônia & 3,868 & 2,$913 ; 5,135$ & $<0,0001$ \\
\hline Santa Catarina & 4,315 & 3,$306 ; 5,631$ & $<0,0001$ \\
\hline São Paulo & 5,694 & 4,$385 ; 7,396$ & $<0,0001$ \\
\hline Sergipe & 5,827 & 4,$451 ; 7,627$ & $<0,0001$ \\
\hline Tocantins & 3,100 & 2,$300 ; 4,180$ & $<0,0001$ \\
\hline Outros (Acre + Amapá + Roraima) & 5,332 & 4,$036 ; 7,043$ & $<0,0001$ \\
\hline
\end{tabular}

IC95\%: intervalo de 95\% de confiança; RP: razão de prevalência.

custo” na época em estudo. Naquele momento, ainda não havia clara definição das responsabilidades de cada gestor - federal, estadual e municipal 23. Dentro da lógica de cofinanciamento dos medicamentos do Componente Especializado de Assistência Farmacêutica 23, o percentual encontrado de pacientes em uso de eritropoetina também poderia significar subnotificação de APAC por parte dos estados. Em outras palavras, no período estudado, os estados poderiam estar assumindo uma parcela importante da aquisição da eritropoetina para seus pacientes, sem 
informar à União, para que o repasse financeiro fosse realizado.

O percentual de pacientes que fizeram suplementação de ferro III (18\%) também está aquém do esperado. Dados do Censo de Diálise $2008{ }^{3}$ registram uso de ferro III por $53,5 \%$ dos pacientes em tratamento dialítico. Tal como no caso da utilização da eritropoetina, as considerações feitas em relação às mudanças decorrentes da reforma da política assistencial 23 são pertinentes. Outra hipótese seria um possível subtratamento da população estudada. Considerando a importância da suplementação de ferro para pacientes com deficiência desse mineral, a fim de garantir a efetividade da eritropoetina 6 , se comprovado o subtratamento, tem-se um achado preocupante, já que poderia indicar problemas no manejo correto da anemia em pacientes em hemodiálise, conforme relatado por Richardson et al. ${ }^{11}$.

No que se refere ao perfil da coorte geral e ao perfil dos pacientes segundo o uso de eritropoetina, o predomínio do sexo masculino é condizente com os achados de Acurcio et al. 17, Cherchiglia et al. 18 e de Sesso et al. 3 , que relataram predomínio dos homens entre os pacientes em tratamento de doença renal crônica no Brasil. Achados de Locatelli et al. 10 também registraram predomínio de homens em tratamento dialítico nos países europeus, com proporções maiores de homens entre os pacientes incidentes, comparados aos prevalentes.

Os pacientes que utilizaram eritropoietina mostraram-se, em média, mais jovens do que os da coorte estudada e do que os que não utilizaram o medicamento. Maddux et al. 19, examinando dados de seguros de saúde norte-americanos, referentes a pacientes com doença renal crônica maiores de 15 anos, também observaram menor percentual de utilização de eritropoetina por pacientes acima de 65 anos. Esse achado pode refletir deficiências no cuidado destinado à saúde da população idosa, com subtratamento da anemia neste grupo, e fragilidades relativas aos fluxos de referência dos programas de assistência farmacêutica no período estudado.

A maior concentração de pacientes que utilizaram eritropoetina na Região Sudeste e no Nordeste está de acordo com os achados para a população em terapia renal substitutiva no Brasil, conforme descrito por Cherchiglia et al. 18 , de Moura et al. 24 e Sesso et al. ${ }^{3}$, correspondendo ao esperado para a população brasileira. Notase que a distribuição dos pacientes por região, segundo o uso de eritropoetina, reflete as desigualdades regionais, considerando-se a extensão territorial do Brasil; reflete, ainda, as iniquidades em relação à política de atenção ao portador de doença renal crônica e às variações em relação à execução da política de assistência farmacêutica nas regiões e também nos estados 23,25,26.

Outro achado interessante foi a maior proporção de diabetes mellitus e de glomerulonefrites como causas primárias de doença renal crônica entre os pacientes em uso de eritropoetina, quando comparados com o grupo que não utilizou o medicamento. Abaterusso et al. 27 conduziram uma revisão da literatura sobre a anemia em pacientes diabéticos, definindo-a como mais frequente e mais severa do que a ocorrida em pacientes com outras causas de doença renal crônica. Isto explicaria a maior participação dos diabéticos no grupo que utilizou eritropoetina, denotando também melhor seguimento desta subpopulação. A proporção relevante de pacientes com glomerulonefrites, as quais podem estar relacionadas a agentes infecciosos, demonstra que, no Brasil, deparamos com características dos processos da "transição epidemiológica” observada mundialmente, verificando-se crescimento da morbimortalidade por doenças crônicas não transmissíveis e ocorrência ainda elevada das doenças infecciosas 28 .

O maior percentual de pacientes iniciando hemodiálise com fístula arteriovenosa no grupo que utilizou eritropoetina, em comparação com o grupo que não utilizou o medicamento e também com a coorte global, embora pareça ser um indicador de melhor seguimento terapêutico dos pacientes que utilizam eritropoetina, ainda está longe do ideal e, mesmo, da realidade de outros países 29 .

A proporção de óbitos do grupo que utilizou eritropoetina foi relativamente pequena em relação à população geral estudada e bem menor do que a observada para o grupo que não utilizou o fármaco. Esse quadro pode confirmar a contribuição do tratamento da anemia para a diminuição da mortalidade nos pacientes em hemodiálise, conforme relatado na literatura 4,9,12,13.

Houve maior percentual de pacientes que fizeram suplementação de ferro III no grupo que utilizou eritropoetina. Esse achado é condizente com os protocolos instituídos para tratamento da anemia, os quais recomendam a suplementação de ferro para pacientes que apresentem depleção orgânica das reservas do mineral, detectada laboratorialmente. Tal conduta garante a efetividade da eritropoetina na correção da anemia na doença renal crônica 6,10,12,30.

Entre os pacientes que fizeram uso da eritropoetina houve maior percentual de transplantados. Esse resultado pode significar que os pacientes cuja anemia é tratada com eritropoetina estão em melhores condições clínicas e, portanto, mais aptos a realizar transplantes. O tratamento da anemia baseado em transfusões sanguíneas, 
além de expor o paciente à transmissão de agentes infecciosos, caso um doador infectado esteja em "janela imunológica”, pode favorecer a rejeição do enxerto 5 .

A maioria dos pacientes, independentemente de usar ou não eritropoetina, fez seu tratamento no serviço privado, significando que o SUS, apesar de ser o principal financiador das terapias renais, não é o principal prestador de serviços, contratando serviços de prestadores privados.

Verificou-se, neste estudo, que o sexo masculino apresentou maior razão de prevalência de uso de eritropoietina do que o feminino. Do ponto de vista clínico, este é um achado surpreendente. A anemia tende a ser mais severa em pacientes do sexo feminino, fazendo com que utilizem eritropoetina e suplementos de ferro mais frequentemente, necessitando, também, de doses elevadas desses medicamentos 19,31. Entretanto, o estudo realizado por Acurcio et al. 17, sobre o perfil demográfico e epidemiológico de usuários de medicamentos de alto custo no Brasil, relatou maior utilização de eritropoetina pelos homens, que também são a maioria dos pacientes com doença renal crônica. Análise do perfil dos pacientes estratificada por sexo, em nossa investigação, demonstrou que o sexo feminino teve menor tempo médio de acompanhamento, além de maior percentual de óbitos. As diferenças encontradas entre os sexos, para estas variáveis, foram estatisticamente significativas (dados não mostrados). Portanto, muito mais do que uma questão clínica, o resultado encontrado pode significar iniquidade no acesso ao cuidado e aos medicamentos, segundo o sexo dos pacientes.

Maior razão de prevalência para uso de eritropoetina também esteve associada a pacientes com idade inferior 65 anos, achado semelhante ao de Maddux et al. 19. Um estudo brasileiro que abordou o acesso a medicamentos para doenças crônicas por pacientes adultos e idosos no Nordeste e no Sul do Brasil identificou que a escolaridade, um indicador socioeconômico, influenciou no acesso da população idosa aos medicamentos nas duas regiões 24 , enfatizando a iniquidade de acesso para a população em análise. Nosso estudo aponta uma tendência, que merece ser investigada por outras pesquisas, de uma possível iniquidade de acesso da população idosa ao tratamento da anemia da doença renal crônica.

A presença do diabetes mellitus como causa primária da doença renal crônica apresentou maior razão de prevalência para uso de eritropoetina em relação à ausência de diabetes. Este achado é condizente com dados da literatura, que apontam que os pacientes com diabetes mellitus têm anemia mais severa, necessitam de doses mais elevadas do fármaco e têm maior risco de morte do que os pacientes com outras causas de doença renal crônica 27,31 . O resultado encontrado também sugere melhor seguimento terapêutico dos pacientes com nefropatia diabética, quando comparados aos pacientes com doença renal crônica por outras causas, a exemplo do relatado por Lee et al. 32 .

A maior razão de prevalência de uso de eritropoetina decorrente de iniciar hemodiálise com fístula arteriovenosa foi um resultado diferente do esperado, considerando-se a literatura. Pesquisas mostram que pacientes que utilizam cateter em vez de fístula apresentam anemia mais severa e necessitam de doses mais elevadas de eritropoetina 22,31. No nosso estudo, o resultado obtido pode significar que pacientes que têm acesso à eritropoetina têm sido, em geral, pacientes com melhor seguimento terapêutico.

Todas as Unidades Federativas do Brasil apresentaram maior razão de prevalência de uso de eritropoetina do que o Estado do Mato Grosso. Apenas para o Rio Grande do Sul este resultado não foi estatisticamente significativo. Análise descritiva dos pacientes desagregada por estado mostrou que o Mato Grosso apresentou o menor percentual de pacientes transplantados durante o período de acompanhamento. Mato Grosso, Alagoas e Rondônia, nesta ordem, tiveram os menores tempos medianos até o óbito. Mato Grosso e Mato Grosso do Sul, nesta ordem, tiveram os menores tempos de acompanhamento (dados não mostrados). O fato de o Mato Grosso se localizar numa área de fronteira pode sugerir que o gestor estadual tenha dificuldade em implementar uma política de atenção à saúde efetiva, já que a população pode assumir um caráter flutuante. O desmembramento político-jurídico recente do estado do Mato Grosso, em 1977, pode indicar, ainda, que o estado ainda não se estruturou adequadamente como unidade federativa, em relação às políticas públicas. Análise descritiva do Estado do Mato Grosso do Sul, originado do desmembramento do Mato Grosso, igualmente indica fragilidades em relação a aspectos clínicos dos pacientes, com baixo percentual de pacientes recebendo suplementação de ferro e baixo percentual de transplantados (dados não apresentados), sugerindo que, neste estado, como no outro, pudesse haver inadequação das políticas públicas na época analisada.

Goiás e Tocantins, componentes do Estado de Goiás até 1988, também apresentaram diferenças do ponto de vista de utilização de ferro e eritropoetina, ocorrência de óbitos e de transplantados (dados não mostrados), que podem relacionarse ao desmembramento recente e infraestrutura precária como Unidades Federativas. 
O Estado do Amazonas apresentou o maior percentual de pacientes utilizando eritropoetina dentre os estados brasileiros e também a maior razão de prevalência para o uso, em comparação ao Mato Grosso. Considerando o período em que o estudo foi desenvolvido, os resultados parecem indicar que aquele estado dependia fundamentalmente do repasse federal e que o acesso de sua população à eritropoetina se fazia quase que exclusivamente por meio de APAC.

As diferenças entre os estados, aqui ilustradas por alguns exemplos e apresentadas por meio do modelo múltiplo proposto, indicam desigualdades regionais e estaduais no que diz respeito à oferta e à utilização de serviços de saúde 25,26. Especialmente no que se refere à fragilidade da definição das responsabilidades dos gestores, própria do cenário regulatório da época, enfatizam que os fluxos de gerenciamento dos medicamentos de alto custo ou "excepcionais", que atualmente integram o Componente Especializado de Assistência Farmacêutica, apresentavam ampla variação no Brasil, conforme documentado pelo Ministério da Saúde em publicação recente 23.

O presente trabalho possui algumas limitações. Por se tratar de um estudo transversal, relações de causa não podem ser inferidas. Os dados da Base Nacional em Terapia Renal Substitutiva são provenientes de bases administrativas, cujo propósito é o faturamento e não a pesquisa. Dessa forma, verificou-se, para muitas variáveis, a ocorrência de informações incompletas, inconsistentes e inexistentes. Por exemplo, pode ter havido sub-registro de informações importantes, incluindo o uso de medicamentos, e de dados clínicos dos pacientes, como verificado nos estudos de Acurcio et al. 17, de Moura et al. 24 e de Cherchiglia et al. 18 , todos conduzidos valendo-se de dados administrativos. Além disso, notou-se ausência de informações complementares para avaliação da anemia, tais como dados clínicos (níveis de hemoglobina e hematócrito, ao longo do tempo de acompanhamento, comorbidades e dados de hospitalização) e outros dados demográficos (raça, escolaridade e renda).

\section{Conclusão}

Os resultados deste estudo reforçam a contribuição da eritropoetina na redução da mortalidade em pacientes portadores de doença renal crônica. Porém, a utilização deste medicamento para anemia na doença renal crônica parece estar relacionada a pacientes com melhor seguimento terapêutico e melhores condições clínicas. Trata-se de um achado preocupante, na medida em que pacientes em pior estado de saúde, que necessitariam de uma abordagem terapêutica mais intensiva, podem estar deixando de ter acesso ao medicamento simplesmente porque vão a óbito antes mesmo de terem oportunidade de utilizá-lo.

Os resultados indicam iniquidades no acesso considerando o sexo feminino, idade maior que 65 anos, presença de nefropatia diabética, acesso vascular inicial na hemodiálise e fragilidades estruturais em estados com menor organização política e econômica. Por isso, a política de atenção ao portador de doença renal crônica e a forma de alocação de recursos para os estados e municípios devem ser periodicamente revistas, de forma a garantir que a obtenção dos medicamentos essenciais ao tratamento da doença renal crônica e suas complicações se dê segundo os princípios da equidade e da universalidade do acesso.

Por outro lado, grandes avanços foram obtidos na política de assistência farmacêutica, tais como a revisão recente do protocolo brasileiro de tratamento da anemia na doença renal crônica, em 2010 2,6, e uma melhor definição das responsabilidades dos três níveis de gestão do SUS, por meio da Portaria no. 2.981/2009 14,23. E é imperativo que eles continuem. Há necessidade de avaliação constante do impacto dos marcos regulatórios, identificando falhas e corrigindoas. Além disso, o manejo da anemia, segundo o perfil farmacoterapêutico dos pacientes, levando em conta suas características peculiares, deve ser enfatizado, a fim de garantir a redução das iniquidades apresentadas e a concordância com outro princípio doutrinário do SUS: a integralidade da atenção. 


\section{Resumo}

Este estudo visa a descrever o perfil demográfico e epidemiológico de pacientes incidentes em hemodiálise, que utilizaram eritropoetina em 2002 e 2003, no Brasil, e identificar fatores associados ao uso desse medicamento. Foram analisados dados demográficos, clínicos e relacionados à unidade de diálise de 32.136 pacientes, identificados por pareamento determinísticoprobabilístico de dados do subsistema de autorização de procedimentos de alto custo e do Sistema de Informações sobre Mortalidade. Um modelo de regressão de Poisson foi usado para identificar fatores associados ao uso de eritropoetina. Sexo masculino; idade inferior a 65 anos; nefropatia diabética; fístula arteriovenosa ao iniciar hemodiálise e residir em outras Unidades Federativas diferentes do Mato Grosso foram fatores associados ao uso de eritropoetina. A política de atenção ao doente renal, a alocação de recursos para estados e municípios e o manejo da anemia, segundo o perfil farmacoterapêutico dos pacientes, devem ser revistos, a fim de reduzir as iniquidades observadas na utilização da eritropoetina.

Eritropoetina; Diálise Renal; Anemia; Equidade no Acesso

\section{Colaboradores}

T. C. Gurgel, M. L. Cherchiglia, F. A. Acurcio, D. A. C. Szuster e E. I. G. Andrade, participaram da concepção, planejamento, desenho, análise e interpretação dos dados, revisão crítica do conteúdo e aprovação da versão final do manuscrito. I. C. Gomes colaborou na análise e interpretação dos dados, revisão crítica do conteúdo e aprovação da versão final do manuscrito

\section{Agradecimentos}

Os autores agradecem aos demais pesquisadores do Grupo Pesquisa em Economia da Saúde da Universidade Federal de Minas Gerais, por sugestões ao projeto e ao manuscrito; ao Conselho Nacional de Desenvolvimento Científico e Tecnológico (CNPq), à Fundação de Amparo à Pesquisa do Estado de Minas Gerais (FAPEMIG) e ao Ministério da Saúde, pelo apoio financeiro.

\section{Referências}

1. Bastos MG, Carmo WB, Abrita RR, Almeida EC, Mafra D, Costa DMN, et al. Doença renal crônica: problemas e soluções. J Bras Nefrol 2004; 26 : 202-15.

2. Harrison TR, Fauci AS. Medicina interna. 16a Ed. Rio de Janeiro: McGraw-Hill; 2006.

3. Sesso R, Lopes AA, Thomé FS, Bevilacqua JL, Romão Jr. JEJ, Lugon J. Relatório do Censo Brasileiro de Diálise, 2008. J Bras Nefrol 2008; 30:233-8.

4. Bastos MG. Anemia e progressão da doença renal crônica. J Bras Nefrol 2006; 28(3 Suppl 2):S18-21.

5. Duh MS, Weiner JR, White LA, Lefebvre P, Greenberg PE. Management of anaemia. A critical and systematic review of the cost effectiveness of erythropoiesis-stimulating agents. Pharmacoeconomics 2008; 26:99-120.

6. Ministério da Saúde. Portaria SAS/MS no. 226, de 10 de maio de 2010. Aprova o protocolo clínico e diretrizes terapêuticas da anemia na insuficiência renal crônica. Diário Oficial da União 2010; 11 mai.
7. Ammirati AL, Watanabe R, Aoqui C, Draibe SA, Carvalho AB, Abensur H, et al. Variações nos níveis de hemoglobina de pacientes em hemodiálise tratados com eritropoietina: uma experiência brasileira. Rev Assoc Med Bras 2010; 56:209-13.

8. McClellan W, Aronoff, SL, Bolton WK, Hood S, Lorber DL, Tang KL, et al. Prevalence of anemia in patients with chronic renal disease. Curr Med Res Opin 2004; 20:1501-10.

9. Collins AJ, Brenner RM, Ofman, JJ, Chi EM, Stuccio-White N, Krishnan M, et al. Epoetin alfa use in patients with ESRD: an analysis of recent US prescribing patterns and hemoglobin outcomes. Am J Kidney Dis 2005; 46:481-8.

10. Locatelli F, Pisoni RL, Combe C, Bommer J, Andreucci VE, Piera L, et al. Anaemia in haemodialysis patients of five European countries: association with morbidity and mortality in the Dialysis Outcomes and Practice Patterns Study (DOPPS). Nephrol Dial Transplant 2004; 19:121-32. 
11. Richardson D, Hodsman A, van Schalkwyk D, Tomson C, Warwick G. Management of anaemia in haemodialysis and peritoneal dialysis patients (chapter 8). Nephrol Dial Transplant 2007; 22 Suppl 7:vii78-104.

12. Canziani MFE, Bastos MG, Bregman R, Pecoits Filho R, Tomiyama CM, Draibe AS, et. al. Deficiência de ferro e anemia na doença renal crônica. J Bras Nefrol 2006; 28:86-90.

13. Thorp ML, Johnson ES, Yang X, Petrik AF, Platt R, Smith DH. Effect of anaemia on mortality, cardiovascular hospitalizations and end-stage renal disease among patients with chronic kidney disease. Nephrology 2009; 14:240-6.

14. Ministério da Saúde. Portaria GM/MS nº. 2981, de 26 de novembro de 2009. Aprova o Componente Especializado de Assistência Farmacêutica. Diário Oficial da União 2010; 30 nov.

15. Coyne DW. Managing anemia in for-profit dialysis chains: when ethics and business conflict. Semin Dial 2009; 22:18-21.

16. Locatelli F, Covic A, Kai-Uwe E, Wiecek A, Vanholder R. Anaemia management in patients with chronic kidney disease: a position statement by the Anaemia Working Group of European Best Practice (ERBP). Nephrol Dial Transplant 2009; 24:348-54.

17. Acurcio FA, Brandão CMR, Guerra Jr. AA, Cherchiglia ML, Andrade EIG, Almeida AM, et al. Perfil demográfico e epidemiológico dos usuários de medicamentos de alto custo no Sistema Único de Saúde. Rev Bras Estud Popul 2009; 26:263-82.

18. Cherchiglia ML, Machado EL, Szuster DAC, Andrade EIG, Acurcio FA, Caiaffa WT, et al. Perfil epidemiológico dos pacientes em terapia renal substitutiva no Brasil, 2000-2004. Rev Saúde Pública 2010; 44:639-49.

19. Maddux FW, Shetty S, Del Aguila MA, Nelson MA, Murray BM. Effect of erythropoiesis-stimulating agents on healthcare utilization, costs and outcomes in chronic kidney disease. Ann Pharmacother 2007; 41:1761-9.

20. Cherchiglia ML, Guerra Jr. AA, Andrade EIG, Machado CJ, Acurcio FA, Meira Jr. W, et.al. A construção da base de dados nacional em terapia renal substitutiva (TRS) centrada no indivíduo: aplicação do método de linkage determinístico-probabilístico. Rev Bras Estud Popul 2007; 24:163-7.

21. Programa das Nações Unidas para o Desenvolvimento. Atlas do desenvolvimento humano no Brasil. Brasília: Programa das Nações Unidas para o Desenvolvimento; 2000.

22. Pérez-García R, Martín-Malo A, Fort J, Cuevas X, Lladós F, Lozano J, et al. Baseline characteristics of an incident haemodialysis population in Spain: results from ANSWER - a multicentre, prospective, observational cohort study. Nephrol Dial Transplant 2008; 24:578-88.
23. Ministério da Saúde. Da excepcionalidade às linhas de cuidado: o Componente Especializado de Assistência Farmacêutica. Brasília: Ministério da Saúde; 2010.

24. Moura L, Schmidt MI, Duncan BB, Rosa RS, Malta DC, Stevens A, et al. Monitoramento da doença renal crônica terminal pelo subsistema de Autorização de Procedimentos de Alta Complexidade - APAC - Brasil, 2000 a 2006. Epidemiol Serv Saúde 2009; 18:121-31.

25. Paniz VMV, Fassa AG, Facchini LA, Bertoldi AD, Piccini RX, Tomasi E, et al. Acesso a medicamentos de uso contínuo em adultos e idosos nas regiões Sul e Nordeste do Brasil. Cad Saúde Pública 2008; 24:267-80.

26. Travassos C, Oliveira EXG, Viacava F. Desigualdades geográficas e sociais no acesso aos serviços de saúde no Brasil: 1998 e 2003. Ciênc Saúde Coletiva 2006; 11:975-86.

27. Abaterusso C, Pertica N, Lupo A, Ortalda V, Gambaro G. Anaemia in diabetic renal failure: is there a role for early erythropoietin treatment in preventing cardiovascular mortality? Diabetes Obes Metab 2008; 10:843-9.

28. Malta DC, Cezário AC, Moura L, Morais Neto OL, Silva Jr. JB. A construção da vigilância e a prevenção das doenças crônicas não transmissíveis no contexto do Sistema Único de Saúde. Epidemiol Serv Saúde 2006; 15:47-65.

29. Ethier J, Mendelssohn DC, Elder SJ, Hasegawa T, Akizawa T, Akiba T, et al. Vascular access use and outcomes: an international perspective from the Dialysis Outcomes and Practice Patterns Study. Nephrol Dial Transplant 2008; 23:3219-26.

30. Gilmartin C. Pharmacist's role in managing anemia in patients with chronic kidney disease: Potential clinical and economic benefits. Am J Health Syst Pharmacists 2007; 64(13 Suppl 8):S15-22.

31. Verdalles U, Vinuesa SG, Goicoechea M, Campderá FG, Luño J. Evaluación del cumplimiento de las guias terapéuticas en la práctica clínica en pacientes con enfermedad renal crónica (ERC). Nefrología 2006; 27:320-8.

32. Lee T, Barker J, Allon M. Associations with predialysis vascular access management. Am J Kidney Dis 2004; 43:1008-13.

Recebido em 29/Dez/2010

Versão final reapresentada em 28/Set/2011 Aprovado em 31/Jan/2012 